\title{
An electrical-filtered optical heterodyne technique for tuning speed measurement of DBR lasers
}

\author{
MAN JiangWei, ZHU NingHua*, ZHANG HongGuang, SUN Ke, KE JianHong, HAN Wei, \\ CHEN Wei, LIU Yu, WANG Xin, YUAN HaiQing \& XIE Liang
}

State Key Laboratory on Integrated Optoelectronics, Institute of Semiconductors, Chinese Academy of Sciences, Beijing 100083, China

Received May 20, 2010; accepted July 22, 2010

\begin{abstract}
A new scheme based on the electrical-filtered optical heterodyne technique is proposed for measuring the tuning speed of tunable distributed Bragg reflector (DBR) lasers. In this scheme, a $10 \mathrm{GHz}$ high-pass electrical filter is used and the wavelength switching time of a tunable DBR laser for different tuning frequencies corresponding exactly to different delay lines is measured. The switching time is measured to be nearly $300 \mathrm{~ns}$ and can be improved by reducing the equivalent resistance-capacitance (RC) time constant of the device. The distribution of the beat signal of the DBR laser in the time domain is also obtained, and is a good match with the waveforms measured using an optical filter.
\end{abstract}

DBR laser, electrical filter, square-wave tuning, tuning speed, wavelength switching

Citation: Man J W, Zhu N H, Zhang H G, et al. An electrical-filtered optical heterodyne technique for tuning speed measurement of DBR lasers. Chinese Sci Bull, 2011, 56: 704-708, doi: 10.1007/s11434-010-4160-x

The tunable distributed Bragg reflector (DBR) lasers, with the advantage of fast discreet and continuous tuning speeds on both the Bragg section and the phase section [1], have many applications in frequency-hopping communications, next-generation optical switch fabrics and packet-switched wavelength-division-multiplexed systems. In such systems, the fast wavelength tuning speed of the DBR laser is needed, which is an important parameter that determines the wavelength switching speed among different channels and the minimum guard time among adjacent packets as well as the transport efficiency of the system [2]. The wavelength tuning speed is greatly dependent on the thermal effect induced by the tuning current and can be effectively reduced by a low output-impedance driver. Designing the sampled grating mirrors with reduced peak spacing to limit the tuning current, or tuning DBR lasers using pulse signals with pre-emphasis, can also improve the tuning performance of DBR lasers [3, 4].

To date, many methods for the tuning speed measure-

*Corresponding author (email: nhzhu@semi.ac.cn) ment of tunable DBR lasers have been proposed. A timeresolved spectrum technique based on a scanning FabryPérot interferometer was successfully used in wavelength switching measurements on SG-DBR lasers [5, 6]. In [7, 8], an optical filter was needed to filter out the lightwave in a certain channel, and the optical filter was required to have a tunable central wavelength and good stability. In [9], the authors tracked the wavelength switching behavior of a tunable laser by analyzing the low-frequency beat signal in the time-frequency domain based on a self-heterodyne method. In [10], a fixed-wavelength reference lightwave was employed for time-resolved characteristic measurements of a SG-DBR laser for optical packet switched networks.

In this paper, a new scheme is proposed to measure the tuning speed of DBR lasers using an electrical filtered optical heterodyne technique. In this method, the wavelength switching time is measured under different tuning frequencies and the distribution of the beat signal of DBR lasers in the time domain is also obtained, compared with the waveforms measured using an optical filter. 


\section{Analysis and experimental setup}

The experimental setup for tuning speed measurement of the tunable DBR lasers is presented in Figure 1. In this scheme, a $1.55 \mu \mathrm{m}$ four-section DBR laser is used which includes an active section, a phase section and two shorted Bragg sections. A low-frequency square-wave signal generated by an Agilent 33250 is applied to the phase section of the DBR laser. A $150 \Omega$ resistor is inserted between the square-wave generator and DBR laser to limit the phase section current. Under square-wave tuning with period $2 \tau_{0}$, injecting currents into the phase section change the refractive index, and therefore, the DBR laser will lase at $\lambda_{1}$ and $\lambda_{2}$ with the duration time $\tau_{0}$, corresponding to the high and low voltage levels $V_{\mathrm{L}}$ and $V_{\mathrm{H}}$. Here, the electric field of the lightwave from the DBR laser is written as

$$
E(t)=E_{0} \exp \left[j\left(\int_{0}^{t} \omega(\tau) \mathrm{d} \tau+\varphi(t)\right)\right],
$$

where $E_{0}$ is the amplitude of the electric field, $\omega(\tau)$ is the angular frequency of the lightwave and $\varphi(t)$ describes the laser phase noise. Under tuning by the square-wave signal, the angular frequency $\omega(\tau)$ of the light beam will be $\omega_{1}$ and $\omega_{2}$ alternatively, corresponding to wavelengths $\lambda_{1}$ and $\lambda_{2}$.

$$
\omega(\tau)=\left[\omega_{1} \operatorname{rect}\left(\frac{\tau-\tau_{0} / 2}{\tau_{0}}\right)+\omega_{2} \operatorname{rect}\left(\frac{\tau-3 \tau_{0} / 2}{\tau_{0}}\right)\right] \operatorname{comb}\left(\frac{\tau-\tau_{0}}{2 \tau_{0}}\right),
$$

where the angular frequency of the lightwave can be expressed as the convolution of the sum of two rectangle functions and a comb function. In reality, the lasing wavelength will not switch instantaneously with the injecting currents and a transient time is required. The angular frequency $\omega(\tau)$ is modified as

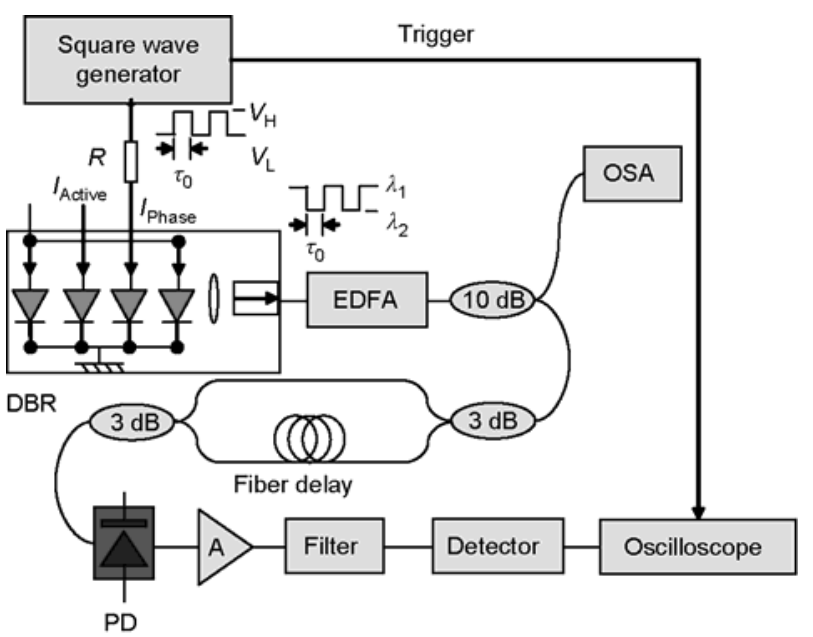

Figure 1 Experimental setup for tuning speed measurement of the tunable DBR-lasers using an electrical filtered optical heterodyne technique. OSA: optical spectrum analyzer, $\mathrm{PD}$ : photodetector, A: microwave amplifier. $\omega(\tau)=\omega_{2} \operatorname{rect}\left(\frac{\tau-\tau_{0} / 2}{\tau_{0}}\right)-\Delta \omega \bullet \operatorname{rect}\left(\frac{\tau-\tau_{0} / 2}{\tau_{0}}\right) \cdot\left[1-\exp \left(-\frac{\tau}{\tau_{1}}\right)\right]$,

$$
\Delta \omega=\omega_{2}-\omega_{1} .
$$

Here the transient time constant is assumed to be $\tau_{1}$ and eq. (3) only gives the angular frequency in a half period for simplicity.

In the experiment, the output lightwave is coupled into a $10 \mathrm{~dB}$ coupler after being amplified by an Er-doped fiber amplifier (EDFA). One part of the lightwave from the $10 \%$ port is used for optical measurement by an optical spectrum analyzer (Advantest Q8384); the other part is launched into an asymmetric Mach-Zehnder interferometer with a delay fiber length corresponding to the delay time $\tau_{0}$ for the optical heterodyne. Thus, the lightwaves with wavelengths $\lambda_{1}$ and $\lambda_{2}$ will arrive at the photodetector (HP 11982A) simultaneously. The total electric field of the lightwaves can be expressed as

$$
E_{\mathrm{total}}=E(t)+E\left(t+\tau_{0}\right) .
$$

Because of the matching of the delay fiber length and the half-period $\tau_{0}$ of the square-wave signal, there is strict correspondence between rise time and fall time in the two channels of the M-Z interferometer which is shown in Figure 2 , and the optical current $i_{\mathrm{PD}}$ generated in the photodetector is written as

$i_{P D} \propto 2 E_{0}^{2} \cos \left[\Delta \omega t+2 \Delta \omega \tau_{1} \exp \left(-\frac{t}{\tau_{1}}\right)-2 \Delta \omega \tau_{1}+\varphi\left(t+\tau_{0}\right)-\varphi(t)\right]$.

During the periods, except for the wavelength switching time, the output signals can beat to generate a microwave signal whose frequency is $\Delta \omega$ corresponding to the wavelength difference between $\lambda_{1}$ and $\lambda_{2}$ at the photodetector, and the noise, whose frequency is less than $\Delta \omega$, also exists with the duration of the switching time between $\lambda_{1}$ and $\lambda_{2}$. After being amplified by an electrical amplifier, the beat signal, whose frequency equals $\Delta \omega$, can pass through, and the noise is filtered out by an electrical filter and the time-varying envelope of the beat signal can be detected by a microwave detector for waveform measurement by an oscilloscope (Rigol DS1204B). Thus, the intensity of the beat signal is not

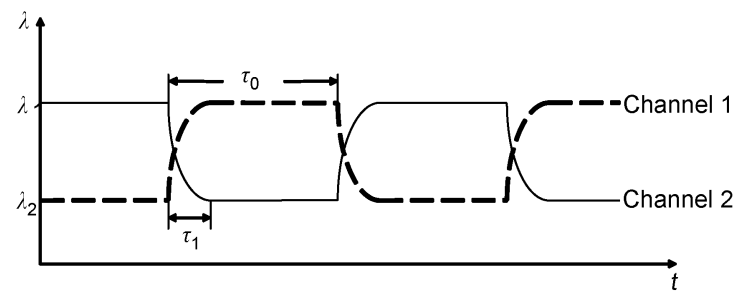

Figure 2 Schematic representation of the Mach-Zehnder interferometer outputs, $\tau_{0}$ : the delay time corresponding to the fiber delay, $\tau_{1}$ : switching time. 
continuous in the time-domain whose period is half that of the square-wave tuning signal and the intervals indicate the wavelength tuning of the DBR laser. Based on the electrical-filtered optical heterodyne technique, the process of wavelength switching has been transferred to the varying intensity detected by the oscilloscope.

\section{Results and discussion}

In the experiment, a tunable DBR laser fabricated in our laboratory was biased at $50.0 \mathrm{~mA}$ in its active section at a constant temperature $25^{\circ} \mathrm{C}$, and the output power is $536 \mu \mathrm{W}$ measured at the pig-tail fiber. The voltage levels $V_{\mathrm{H}}$ and $V_{\mathrm{L}}$ which were applied on its phase section were fixed at 894 $\mathrm{mV}$ and $800 \mathrm{mV}$. Figure 3 shows the measured optical spectrum of the tunable DBR laser under square-wave tuning, the working wavelengths are $1534.440 \mathrm{~nm}$ and $1534.536 \mathrm{~nm}$, corresponding to voltages $V_{\mathrm{H}}$ and $V_{\mathrm{L}}$, with a $0.096 \mathrm{~nm}$ wavelength difference. A $10 \mathrm{GHz}$ high-pass electrical filter was used to filter out the noise generated in the transient time with the high-pass filter transmission shown in Figure 4(c). Figure 4(a) and (b) show the power spectra

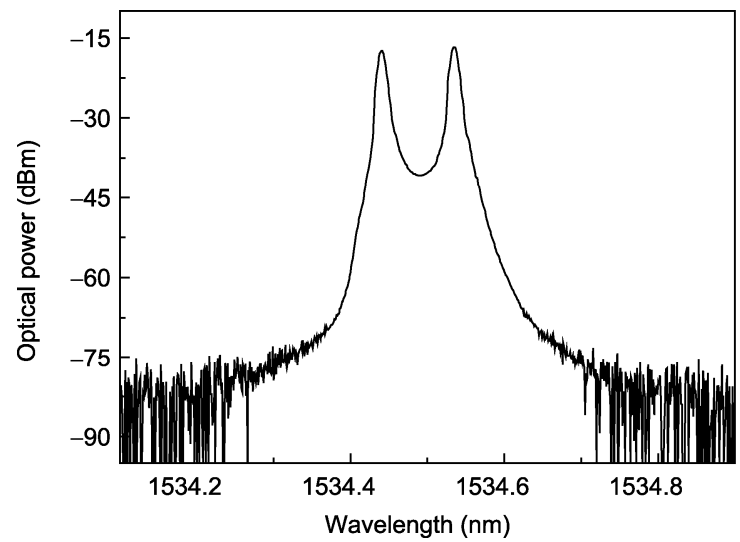

Figure 3 Measured optical spectrum of the tunable DBR laser under square-wave tuning.

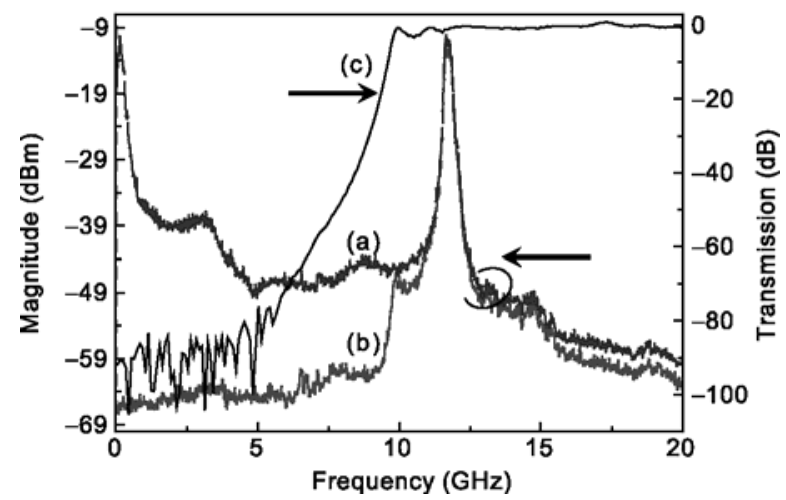

Figure 4 Measured power spectra of the tunable DBR laser under square-wave tuning (a) without electrical filter and (b) with a $10 \mathrm{GHz}$ high-pass filter; (c) measured transmission of a $10 \mathrm{GHz}$ high-pass filter. of the beat signal without and with the electrical filter, which were measured by an electrical spectrum analyzer (Advantest R3182). The noise whose frequency is less than $10 \mathrm{GHz}$ was suppressed by nearly $20 \mathrm{~dB}$ and the beat signal can pass through the filter without any suppression.

Figure 5 shows the square-wave signals applied to the phase section of the DBR laser observed by the oscilloscope. The periods are $100.1300 \mu \mathrm{s}, 4.8294 \mu \mathrm{s}$ and $1.1521 \mu \mathrm{s}$, corresponding to the fiber delay length $10.231 \mathrm{~km}, 493.5 \mathrm{~m}$ and $117.7 \mathrm{~m}$ which were exactly measured by a vector network analyzer (Agilent 8722ET). Figure 6 gives the measured waveforms of the beat signal with different-period square-wave signals applied to the phase section of the DBR laser which was observed in Figure 5. The periods of the beat signal were just half the square-wave periods. From Figure 6 , one can see that the rise time and fall time are asymmetric which is caused by the asymmetry of the switching response time. This is because of the asymmetry of the optical frequency tuning deviation versus the tuning current [1]. It is also limited by the different RC constants of the tuned phase section of the DBR laser in the charging and discharging process. The measured tunable DBR laser took about $300 \mathrm{~ns}$ for the wavelength and optical power of the DBR laser to become steady in each tuning period. With the fiber delay becoming shorter and tuning frequency increasing, the switching time can be measured more precisely. From Figure 5, it can be seen that the rise time of the

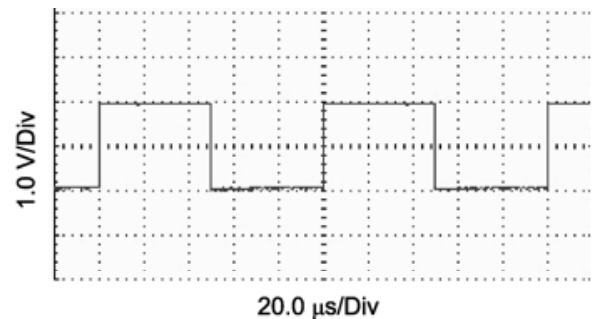

(a)

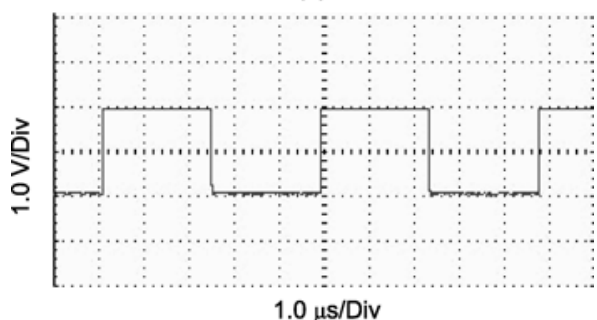

(b)

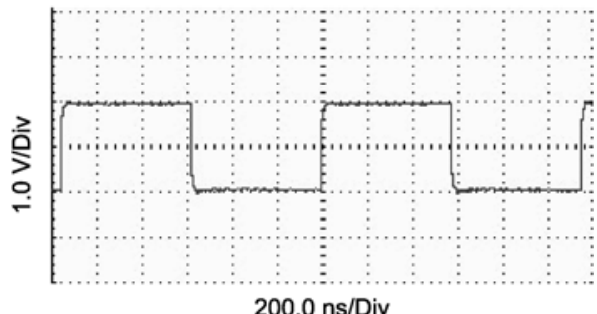

(c)

Figure 5 Square-wave signal applied to the phase section of the DBR laser with different periods. (a) $100.1300 \mu \mathrm{s}$; (b) $4.8294 \mu \mathrm{s}$; (c) $1.1521 \mu \mathrm{s}$. 


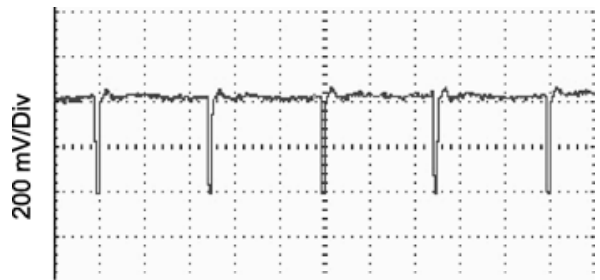

$20.0 \mu \mathrm{s} / \mathrm{Div}$

(a)

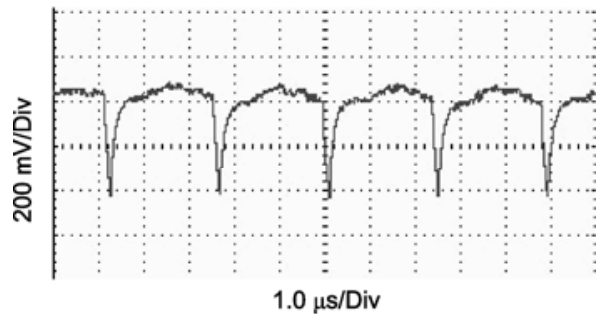

(b)

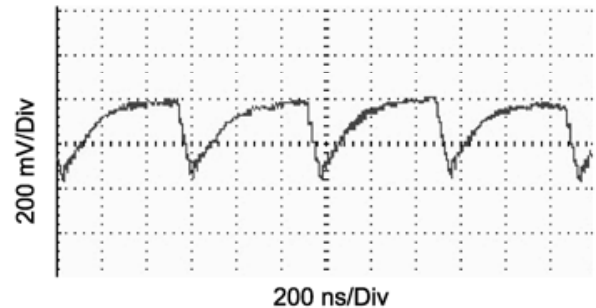

(c)

Figure 6 The measured waveforms of the beat signal with differentperiod square-wave signal applied to the phase section of DBR laser. (a) $100.1300 \mu \mathrm{s}$; (b) $4.8294 \mu \mathrm{s}$; (c) $1.1521 \mu \mathrm{s}$.

square-wave signal applied to the phase section of the DBR laser is about $5 \mathrm{~ns}$; however, the wavelength switching time of the DBR laser is much longer. That is because the wavelength switching time is mostly determined by the heat capacities and thermal resistances of the laser chip, the submount, the heatsink and the thermal contacts [11-13]. On the other hand, the output-impedance of the driver to the phase section strongly affects the wavelength switching time, and the use of a low output-impedance driver to the phase section may reduce the equivalent resistance-capacitance (RC) time constant effectively [2]. With higher current injections into the phase section of the DBR laser, a faster switching time can be achieved at the cost of the greater influence of thermal effects [11]. Using preemphasis at the beginning of the square-wave signal is also a significant way of improving the tuning speed [3].

To confirm the accuracy of the method proposed in Figure 1 , another scheme using an optical filter proposed in [8] was carried out for the same tunable DBR laser under the same tuning period, with the experimental setup shown in Figure 7. In this method, a fiber Bragg grating (JDSU TB9) was used as an optical filter with a full width at half maximum (FWHM) $30 \mathrm{GHz}$ bandwidth. The filter was adjusted to allow only the lightwave with wavelength $\lambda_{1}$ corresponding to the low voltage level $V_{\mathrm{L}}$ to pass through it while the lightwave with wavelength $\lambda_{2}$ was filtered out. Therefore, the lightwave after the filter will be square-wave tuned. As shown in Figure 8, the waveforms measured by the oscilloscope with the method proposed in Figure 7 can be a good match with the former method.

Comparing the electrical-filtered optical heterodyne technique with other methods [5-10], the optical heterodyne technique has the advantages that the choice of the electrical filter is much more arbitrary and the stability of the electrical filter is superior. A tunable optical filter is not necessary and the problems of the instability of the optical filters can be avoided. The limit of the free spectral range of the FabryPérot interferometer would not be encountered. Us-

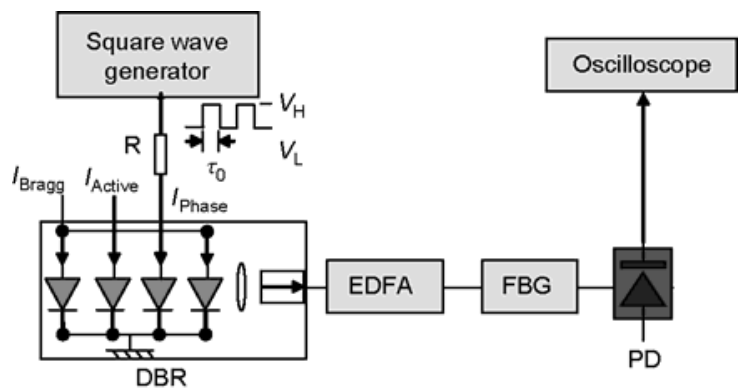

Figure 7 Experimental setup for tuning speed measurement of the tunable DBR lasers using an optical filter. FBG: fiber Bragg grating, PD: photodetector.

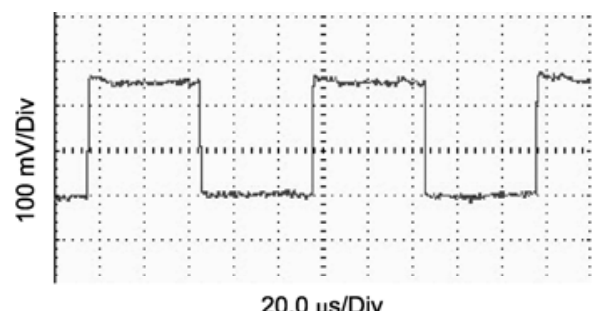

(a)

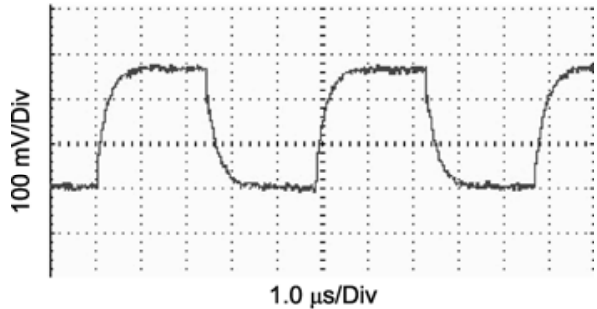

(b)

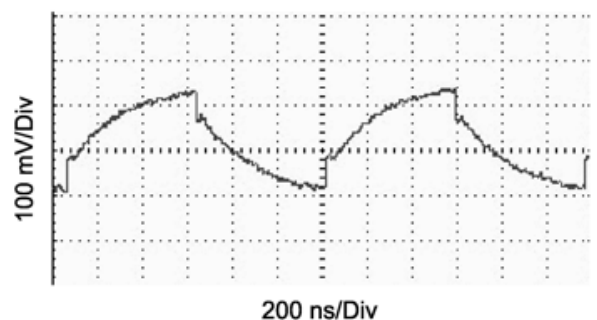

(c)

Figure 8 Measured waveforms in the experimental setup in Figure 7, with different square-wave periods. (a) $100.1300 \mu \mathrm{s}$; (b) $4.8294 \mu \mathrm{s}$; (c) $1.1521 \mu \mathrm{s}$. 
ing the asymmetric Mach-Zehnder interferometer can make the two-wavelength lightwaves arrive at the photodetector simultaneously and another external tunable laser as local oscillator is not required for synchronous detection, which would introduce extra disturbance [14].

\section{Conclusions}

We have proposed a new scheme for tuning speed measurement of the tunable DBR lasers using an electrical filtered optical heterodyne technique. Based on the new scheme, the process of the wavelength switching has been transferred to the varying intensity detected by the oscilloscope. In the measurement, a $10 \mathrm{GHz}$ high-pass filter is used to filter out the noise of the beat signal whose frequency is less than $10 \mathrm{GHz}$. The wavelength switching time is measured at about $300 \mathrm{~ns}$ under different tuning periods corresponding exactly to different delay lines. The wavelength tuning speed can be improved by reducing the heat capacities and thermal resistances of the laser chip, the submount, the heatsink and the thermal contacts. The use of a low output-impedance driver to the phase section may also effectively reduce the equivalent resistance-capacitance (RC) time constant. In this method, a tunable optical filter or a reference lightwave as a local oscillator is not needed and the problem of the instability of the optical filter can be avoided. The choice of the electrical filter is more arbitrary and superior in terms of stability. The distribution of the beat signal of DBR lasers in the time domain is also obtained and is a useful method with a good match to that using an optical filter.

This work was supported by the National Natural Science Foundation of China (60536010, 60606019, 60777029, and 60820106004), the National Basic Research Program of China(2006CB604902, 2006CB302806, 2006dfa11880, and 2009AA03Z409), and the Meteorology Industry Research Project of China (GYHY200806033).

1 Teshima M. Dynamic wavelength tuning characteristics of the
1.5- $\mu \mathrm{m}$ three-section DBR lasers: Analysis and experiment. IEEE J Quantum Electron, 1995, 31: 1389-1400

2 Su Y K, Simsarian J E, Zhang L M. Improving the switching performance of a wavelength-tunable laser transmitter using a simple and effective driver circuit. IEEE Photon Technol Lett, 2004, 16: 2132-2134

3 Rigole P J, Shell M, Nilsson S, et al. Fast wavelength switching in a widely tunable GCSR laser using a pulse pre-distortion technique. In: Stephen A, Joe C, eds. Proceedings of Optical Fiber Communication Conference, 1997 Feb 16-21, Texas. Washington, D C: Optical Society of America, 1997. 231-232

4 Simsarian J E, Larson M C, Garrett H E, et al. Less than 5-ns wavelength switching with an SG-DBR laser. IEEE Photon Technol Lett, 2006, 18: 565-567

5 Davis M, O'Dowd R. Time-resolved spectral measurements on a multielectrode dfb laser using a Fabry-Pérot interferometer. IEEE Photon Technol Lett, 1994, 6: 21-23

$6 \mathrm{Yu} \mathrm{Y,} \mathrm{O'Dowd} \mathrm{R.} \mathrm{Interpretation} \mathrm{of} \mathrm{wavelength} \mathrm{switching} \mathrm{effects} \mathrm{of}$ widely tunable lasers. IEEE Photon Technol Lett, 2002, 14: 1397-1399

7 Bhardwaj A, Gripp J, Simsarian J E, et al. Demonstration of stable wavelength switching on a fast tunable laser transmitter. IEEE Photon Technol Lett, 2003, 15: 1014-1016

8 Zhu N H, Wen J M, San H S, et al. Improved optical heterodyne methods for measuring frequency responses of photodetectors. IEEE J Quantum Electron, 2006, 42: 241-247

9 Joseph H, Sadot D. A novel self-heterodyne method for combined temporal and spectral high-resolution measurement of wavelength transients in tunable lasers. IEEE Photon Technol Lett, 2004, 16: 1921-1923

10 Mishra A, Ellis A, Barry L, et al. Time-resolved linewidth measurements of a wavelength switched SG-DBR laser for optical packet switched networks. In: Loudon B, Joseph E F, eds. Proceedings of Optical Fiber Communication Conference and Exposition and The National Fiber Optics Engineers Conference on CD-ROM, 2008 Feb 24-28, San Diego. Washington, D C: Optical Society of America, 2008. 1-3

11 Braagaard C, Mikkelsen B, Durhuus T, et al. Modelling the dynamics of wavelength tuning in DBR-lasers. IEEE Photon Technol Lett, 1994, 6: 694-696

12 Saavedra A A, Passy R, von der Weid J P. Thermal drift in wavelength-switching DFB and DBR lasers. Electron Lett, 1997, 33: 780-781

13 Mulvihill G, O'Dowd R. Thermal transient measurement, modeling and compensation of a widely tunable laser for an optically switched network. IEEE J Lightw Technol, 2005, 23: 4101-4109

14 Engelstaedter J P, Roycroft B, Peters F H, et al. Heterodyne method for time resolved spectral analysis of fast laser wavelength switching. IEEE Photon Technol Lett, 2009, 21: 1517-1519

Open Access This article is distributed under the terms of the Creative Commons Attribution License which permits any use, distribution, and reproduction in any medium, provided the original author(s) and source are credited. 